\title{
DROPLET SPREADING PROCESS IMPACT ON IGNITION CHARACTERISTICS OF CONDENSED MATERIALS
}

\author{
Gennady Ya. Mamontov ${ }^{1,}$, Galina V. Taratushkina ${ }^{2}$, and Elena Yu. Kurilenko ${ }^{3}$ \\ ${ }^{1}$ National Research Tomsk Polytechnic University, 634050 Tomsk, Russia \\ ${ }^{2}$ Seversk Institute of Technology of MIFI National Nuclear Research University, 636036 Seversk, \\ Russia \\ ${ }^{3}$ Civil engineering Institute of Tyumen Industrial University, 625000 Tyumen, Russia
}

\begin{abstract}
Mathematical simulation of condensed material solid-phase ignition in the context of the in-situ heating by the melted or heated to high temperature metal droplet was carried out. The authors developed the mathematical model that describes the heat transfer process in the "droplet - condensed material" system by the system of heat transfer equations with boundary and initial conditions. The problem is solved by the finite difference method. Four modes of condensed material ignition that are distinguished by the temperature range of every mode were identified for standard conditions of the in-situ heat effect.
\end{abstract}

\section{Introduction}

Ignition processes of condensed materials in the course of the in-situ heating by a single particle have been intensively studied for the last few years. The problems of solid $[1,2]$ and liquid $[3,4]$ fuels are solved. However, the results [1-4] are obtained for conditions when the solid particles had an effect on condensed materials. In the real world, there are some types of liquid-alloy droplet impact on condensed materials. These droplets spread over the cold surface in the course of solidification. Up to the present moment, the problem of fluid drop spreading in the course of its solidification on the cold surface is not solved. Therefore, it is worth analyzing conditions and characteristics of condensed material ignition by a droplet heated to high temperatures while describing the spreading process based on relatively simple models.

\section{Problem statement and solution procedure}

The problem of condensed material ignition by a single hemispherical droplet of a small radius is studied (fig. 1). When the process is simulated, the hemisphere is replaced by a segment with an initial size $h_{\mathrm{o}}$, one edge of which corresponds to the "droplet - condensed material" interface and another one to the droplet upper bound. The type 3 boundary

* Corresponding author: gmamontov@tpu.ru 
conditions that took into consideration the heat transfer with an outdoor environment by means of convection and emission were set at the upper bound. In such a case, the particle spreading was simulated by the hemisphere height change (segment length $h$ ) at a target speed $v$, the value of which was the problem parameter and varied at the rage from 0 to 1.67 $\mathrm{m} / \mathrm{s}$. This range was selected in terms of experimental data on fluid drop spreading speed over the solid surface $[5,6]$.

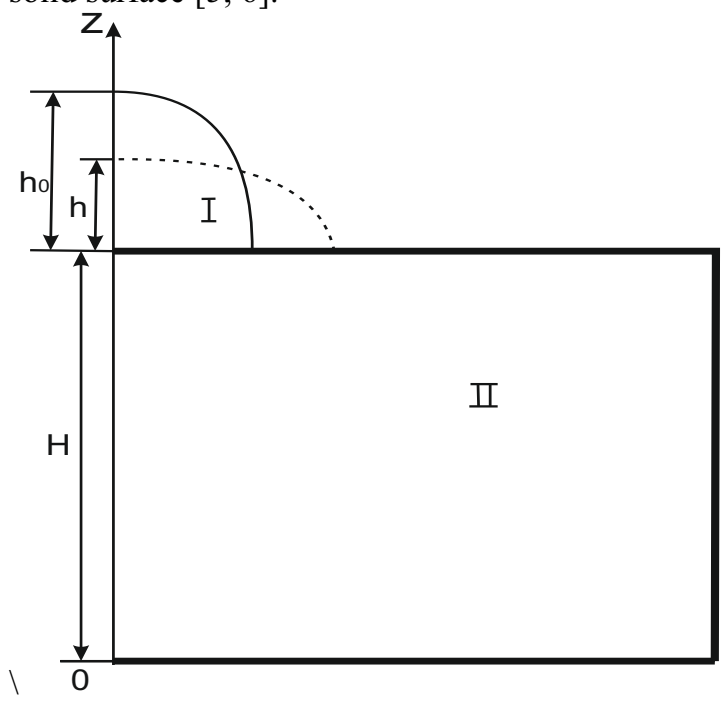

a)

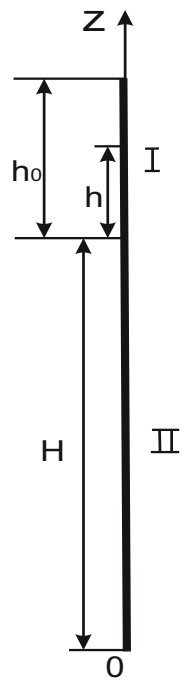

b)

Fig. 1. Problem solution domain: a) geometric interpretation of the drop spreading process; b) stated geometric layout of the process.

The problem was solved in a fixed system of reference. The origin of the system was connected with a nonheating surface of condensed materials. The height of segment I was depressed in the course of spreading. The bed depth of condensed materials was selected in such a way that its initial temperature was preserved on the nonheating surface until the ignition moment. It is determined $[1,2]$ that when the condensed materials are ignited by the single particle heated to high temperatures, the condensed material ignition conditions are first reached on the particle symmetry axis (in axisymmetric problem solving) at the "particle - condensed material" interface point. Hence, the ignition delay times $t_{3}$ obtained in one-dimensional problem solving, at a first approximation can be considered as $t_{3}$ proper values for particles (or droplets) of large diameter (more than $100 \mu \mathrm{m}$ ).

When the problem was set up, it was supposed that the droplet has a temperature $\left(T_{\mathrm{K}}\right)$ that is much higher than an initial temperature $\left(T_{\mathrm{o}}\right)$ of condensed material and an exterior temperature $T_{\mathrm{v}}$ (the process of the melted metal droplet fallback from a "cold" air on a "cold" surface of condensed material was simulated). This set up corresponds to conditions of the hazardous material ignition by melted metal droplets that are formed while cutting off or welding metal structures or in an emergency in the chemical industry when as a result of an explosion the droplets of polymers softened to high temperatures are splashed around the site of explosion.

The problem is set under conditions that when particles do not spread over the condensed material surface, the phase transition heat of metal or polymer solidification is not generated and the steplike thermal and physical characteristics of the droplet material do not change when transferring from one phase state to another. The solid-state reaction of condensed material ignition was studied. 
Under these conditions, the mathematical model of the process under study is a system of equations of the particle energy and the condensed material, and an equation that describes the "response depth" $\eta$ changes under appropriate initial and boundary conditions:

$$
\begin{array}{cc}
\frac{\partial T_{1}}{\partial t}=\frac{\lambda_{1}}{c_{1} \rho_{1}} \frac{\partial^{2} T_{1}}{\partial z^{2}}+\frac{Q}{c_{1}} \frac{d \eta}{d t}, & 0 \leq z \leq H \\
c_{2} \rho_{2} \frac{\partial T_{2}}{\partial t}=\lambda_{2} \frac{\partial^{2} T_{2}}{\partial z^{2}}, & H \leq z \leq H+h \\
\frac{d y}{d x}=k_{0}(1-\eta) \exp \left(-\frac{E}{R T_{1}}\right)
\end{array}
$$

The boundary and initial conditions for the defined problem are:

$$
\begin{array}{cc}
-\lambda_{1} \frac{\partial T_{1}(0, t)}{\partial z}=0, & 0<t<t_{3} \\
-\lambda_{1} \frac{\partial T_{1}(H, t)}{\partial z}=-\lambda_{2} \frac{\partial T_{2}(H, t)}{\partial z}, & T_{1}=T_{2}, \quad 0<t<t_{3} \\
-\lambda_{2} \frac{\partial T_{2}(h+H, t)}{\partial z}=\alpha\left(T_{g}-T_{2}\right)+\varepsilon \sigma\left(T_{g}^{4}-T_{2}^{4}\right), \quad 0<t<t_{3} \\
T_{1}(z, 0)=T_{0}, \quad 0<z<H \\
T_{2}(z, 0)=T_{c h}, \quad H<z<h+H \\
h=h_{0}, \quad t=0 . \\
h=h_{0}-v t, \quad 0<t<t_{3} \\
v=0, T_{2}<T_{\mathrm{pl}} \\
\eta(z, 0)=0, \quad 0<z<H
\end{array}
$$

The numerical analysis was carried out under the following values of the major material and problem parameter characteristics: $\lambda_{1}=0.12 \mathrm{~W} / \mathrm{m} \cdot \mathrm{K} ; \mathrm{c}_{1}=1670 \mathrm{~J} / \mathrm{kg} \cdot \mathrm{K} ; \rho_{1}=500 \mathrm{~kg} / \mathrm{m}^{3}$; $\lambda_{2}=41.0 \mathrm{~W} / \mathrm{m} \cdot \mathrm{K} ; c_{2}=574 \mathrm{~J} / \mathrm{kg} \cdot \mathrm{K} ; \rho_{2}=7870 \mathrm{~kg} / \mathrm{m}^{3} ; \mathrm{E}=111000 \mathrm{~J} / \mathrm{mmol} ; \mathrm{Q}=2.9 \cdot 10^{6} \mathrm{~J} / \mathrm{kg}$; $\mathrm{k}_{0}=7 \cdot 10^{6} 1 / \mathrm{s} ; \varepsilon=0.5 ; \alpha=300 \mathrm{~W} / \mathrm{m}^{2} \mathrm{~K} ; \mathrm{T}_{\mathrm{pl}}=930 \mathrm{~K} ; \mathrm{T}_{0}=\mathrm{T}_{\mathrm{g}}=300 \mathrm{~K}$, where indexes 1 correspond to the condensed material and 2 - to the droplet.

The stated boundary value problem is solved in a similar way $[1,2]$ by the finite difference method using an algorithm developed to solve non-linear problems of the heat transfer with movable boundaries of the simulation domain [7] and in-situ heat release sources [8]. An unequally-spaced and irregular grid [6] was used. The time step was chosen according to conditions of the iteration process convergence [7]. 


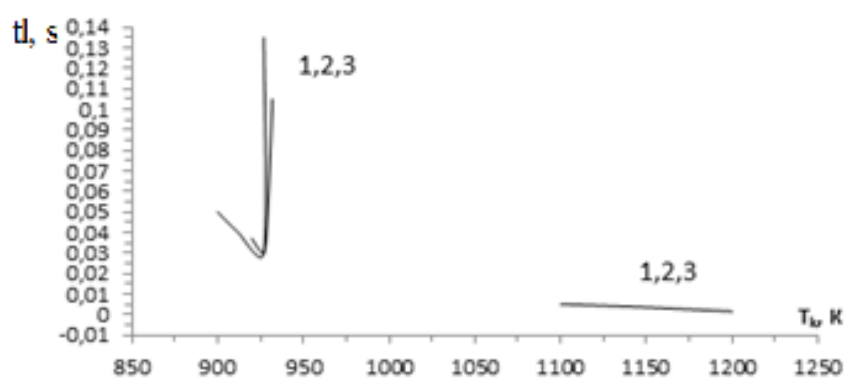

Fig. 2. Dependence of $t_{1}$ on $T_{\mathrm{k}}$ at the "particle lifetime" of $3 \cdot 10^{-3} \mathrm{~s}: 1-h_{0}=0.001 \mathrm{~m}, v=0.333 \mathrm{~m} / \mathrm{s}$; $2-h_{0}=0.003 \mathrm{~m}, v=1.0 \mathrm{~m} / \mathrm{s} ; 3-h_{0}=0.005 \mathrm{~m}, v=1.667 \mathrm{~m} / \mathrm{s}$.

Figure 2 shows ignition delay time dependences of the studied pattern condensed material on the droplet initial temperature under various spreading rates $v$ and the initial droplet height $h_{0} . v$ and $h_{0}$ values were chosen to provide the constant droplet "lifetime" (i.e. time when the droplet height significantly differs from 0 ). Figure 2 displays that it is possible to distinguish four characteristic variation ranges of the droplet initial temperature that correspond to various types of condensed material ignition mechanisms.

The first is a lower temperature range (lower than the droplet metal melting point) that is characterized by $t_{3}$ value reduction when $T_{\mathrm{K}}$ grows and it corresponds to the general requirements of condensed material ignition theory under the in-situ heating $[9,10]$. At this range, the droplet is solid and when $T_{\mathrm{K}}$ grows, the ignition delay time is reduced.

The second is a narrow range where $T_{\mathrm{K}}>T_{\mathrm{pl}}$ and the condensed material ignition process is carried out under conditions of droplet spreading over the condensed material surface. In such a case, due to the high spreading rates, $T_{\mathrm{K}}$ value increase (at the narrow range) causes a paradoxical result - $t_{3}$ increase due to the droplet initial temperature growth. In comparison with the first range, $t_{3}$ change is caused by the fact that under $T_{\mathrm{ch}}>T_{\mathrm{pl}}$ the droplet of a certain height is solidified, and its bigger part spreads over the condensed material surface. Hence, the per-unit-volume heat content (per unit surface area) of the droplet is essential in comparison with the case when the droplet is solid. Thus, the higher the droplet initial temperature is, the bigger the area where it spreads is. In this case, after a complete solidification, the droplet height is lower and more time is necessary to heat the condensed material near-surface layer to meet the ignition requirements.

The reasonable progress of the second range is the third one when the droplet temperature is high enough and decreases quickly when the droplet spreads over the condensed material surface up to the complete solidification. In this case, the spreading time is much lower than the ignition delay time (the solidified layer height is very small, and the droplet is not able to heat the condensed material at a necessary depth to ignite it). If $T_{\mathrm{K}}$ increases, the smaller part of the droplet is left in each section under consideration after the solidification and the heat current at the reaction zone is decreased.

When $T_{\mathrm{K}}\left(T_{\mathrm{K}}<1100 \mathrm{~K}\right)$ values are high enough, the droplet heat content is sufficient to meet the ignition requirements with very low $t_{3}$ values (fig.2). At this range (that can be called the fourth one), the high temperatures of the low height droplet are sufficient and the droplet is able to serve as an in-situ condensed material ignition source.

Based on the obtained results it is possible to make a conclusion that if the droplet "lifetimes" are equal on the condensed material surface, the dependence $t_{3}\left(T_{\mathrm{K}}\right)$ is almost identical (fig.2). In other words, when the droplet spreads over the condensed material surface, $v$ is also a key parameter as well as $T_{K}$.

The specified conformities become apparent when the spreading rates are completely different, $v$ change from $0.333 \mathrm{~m} / \mathrm{s}$ to $1.667 \mathrm{~m} / \mathrm{s}$ does not provoke any remarkable changes 
in $t_{3}$. The ignition condition analysis at high rates $v$ is inappropriate because at high impact rates, even the small droplets will splash and the probability of the condensed material ignition will be close to 0 . Furthermore, it is possible to mention that under the real firehazardous conditions of metal welding or cutting off, the melted metal droplets splash from the ignition zone with the initial rate that is not higher than several dozens of meters per second. Then, the droplet rate dramatically falls.

In conclusion, it is worth mentioning that all obtained results refer to the nondegenerated droplet existence modes on the condensed material surface (for modes when at least $10 \%$ of droplet metal is free of distortion). If the impact velocity is that that the particle spreads immediately up to the thickness of micron units, the condensed material ignition is not possible. When the droplet material melting point rises, the first, the second and the third ignition ranges (fig. 2) are degenerated as at high $T_{\mathrm{K}}$ the ignition delay time of the condensed material will not depend on $T_{\mathrm{K}}$. However, when $T_{\mathrm{K}}$ decreases, the ignition mechanism becomes more complex (fig. 2).

\section{Conclusion}

The results of the carried theoretical studies show the condensed material ignition process in the context of the in-situ melted metal droplet heating and show the ability to realize four mechanisms to initiate the combustion reaction at different droplet temperatures. The received theoretical results and the stated conformities are the basis to develop the condensed material ignition models.

\section{Acknowledgments}

The work was supported by the Russian President's grant (Scientific School project 7538.2016.8).

\section{References}

1. Yu.A. Chumakov, A.G. Knyazeva, Combust. Explos. Shock Waves 48 (2012)

2. G.V. Kuznetsov, G.Ya. Mamontov, G.V. Taratushkina. Khim. Fiz. 23 (2004)

3. G.V. Kuznetsov, P.A. Strizhak., J. Eng. Thermophys. 17 (2008)

4. O.V. Vysokomornaya, G.V. Kuznetsov, P.A. Strizhak., Russ. J. Phys. Chem. B 5 (2011)

5. V.E. Nakoryakov, S.Y. Misyura, S.L. Elistratov. Boiling crisis in droplets of ethanol water solution on the heating surface, J. Eng. Thermophys. 22, 1-7 (2013)

6. V.E. Nakoryakov, S.Y. Misyura, S.L. Elistratov., Int. J. En. Tech. 4 (2012)

7. V.L. Strakhov, A.N. Garashchenko, G.V. Kuznetsov, V.P. Rudzinskii., Combust. Explos. Shock Waves 37 (2001)

8. G.V. Kuznetsov, M.A. Sheremet., Fl. Dyn. 41 (2006)

9. R.S. Burkina, A.G. Knyazeva., Combust. Explos. Shock Waves 28 (1992)

10. G.V. Kuznetsov, G.Ya Mamontov, G.V. Taratushkina., Combust. Explos. Shock Waves. 40 (2004) 